\begin{tabular}{|c|l|}
\hline Title & Chaotic Itinerancy \\
\hline Author(s) & Kaneko, Kunihiko; Tsuda, Ichiro \\
\hline Citation & $\begin{array}{l}\text { Chaos, 13(3), 926-936 } \\
\text { https://doi.org/10.1063/1.1607783 }\end{array}$ \\
\hline Issue Date & 2003-08 \\
\hline Doc URL & http://hdl.handle.net/2115/8486 \\
\hline Type & article (author version) \\
\hline File Information & ci-focus.preface.pdf \\
\hline
\end{tabular}

Instructions for use 


\title{
Chaotic Itinerancy
}

\author{
Kunihiko Kaneko \\ Department of Pure and Applied Sciences \\ University of Tokyo, Komaba, Meguro-ku, Tokyo 153, JAPAN \\ and \\ Ichiro Tsuda \\ Department of Mathematics \\ Hokkaido University, 060-0810, JAPAN
}

July 24, 2003

\begin{abstract}
Chaotic itinerancy is universal dynamics in high-dimensional dynamical systems, showing itinerant motion among varieties of lowdimensional ordered states through high-dimensional chaos. Discovery, basic features, characterization, examples, and significance of chaotic itinerancy are surveyed.
\end{abstract}

About a decade ago, chaotic itinerancy was proposed as a universal dynamical concept in high-dimensional dynamical systems. This was based on numerical studies in coupled maps, optical turbulence, and neural dynamics. One of the great surprises in "deterministic chaos" was the emergence of essential random behavior even in low-dimensional dynamical systems. In the study of highdimensional chaos, however, it was soon recognized that there is often a state that switches back and forth between fully developed chaos and ordered behavior. Here fully developed chaos can be approximated by 'random motion' which may be described as the 
motion consisting of many degrees of freedom, and ordered behavior can often be characterized by low-dimensional dynamics. On the other hand, itinerant motion among varieties of ordered states through high-dimensional chaotic motion is commonly observed. The term for this is chaotic itinerancy.

During the past ten years, chaotic itinerancy has been suggested to be relevant to optical turbulence, protein folding, coupled dynamical systems including globally coupled maps, dynamics of water molecules, climate dynamics, population dynamics in ecosystem, biochemical reaction dynamics in a cell, dynamic memory in human and animal brain, among other topics. Applications of chaotic itinerancy to dynamic control in robotics and to combinatorial optimization problems have also been proposed. Recently, mathematical foundation of the concept has been studied and has developed especially in some ideal cases.

We will summarize the current status of the study of chaotic itinerancy including its recent developments in this interdisciplinary field and also provide a future scope in high-dimensional dynamical systems.

\section{Discovery of chaotic itinerancy}

Chaotic itinerancy (CI) was independently discovered in a model of optical turbulence (by Ikeda) [1], in a globally coupled chaotic system (by Kaneko) [4, 5], and in nonequilibrium neural networks (by Tsuda) [2, 3], and was proposed with unanimous cooperation as universal dynamics in a class of high-dimensional dynamical systems. In CI, an orbit successively itinerates over ordered motion expressed by a few effective degrees of freedom. Considering attraction to, and the residence at the ordered motion state, we called each of such states "attractor-ruin". The motion at "attractor-ruins" is quasi-stationary in the sense that it is close to that in low-dimensional attractor.

After staying at one attractor-ruin, the orbit eventually exits from it. This escape from an attractor-ruin stems from instability of the ruin. (see Fig.1 for schematic representation).

For example, if the effective degrees of freedom is two, the dynamics are in the vicinity of two-dimensional subspace in the original high-dimensional 


\section{Schematic Representation of Chaitic Itinrenacy \\ in phase space}

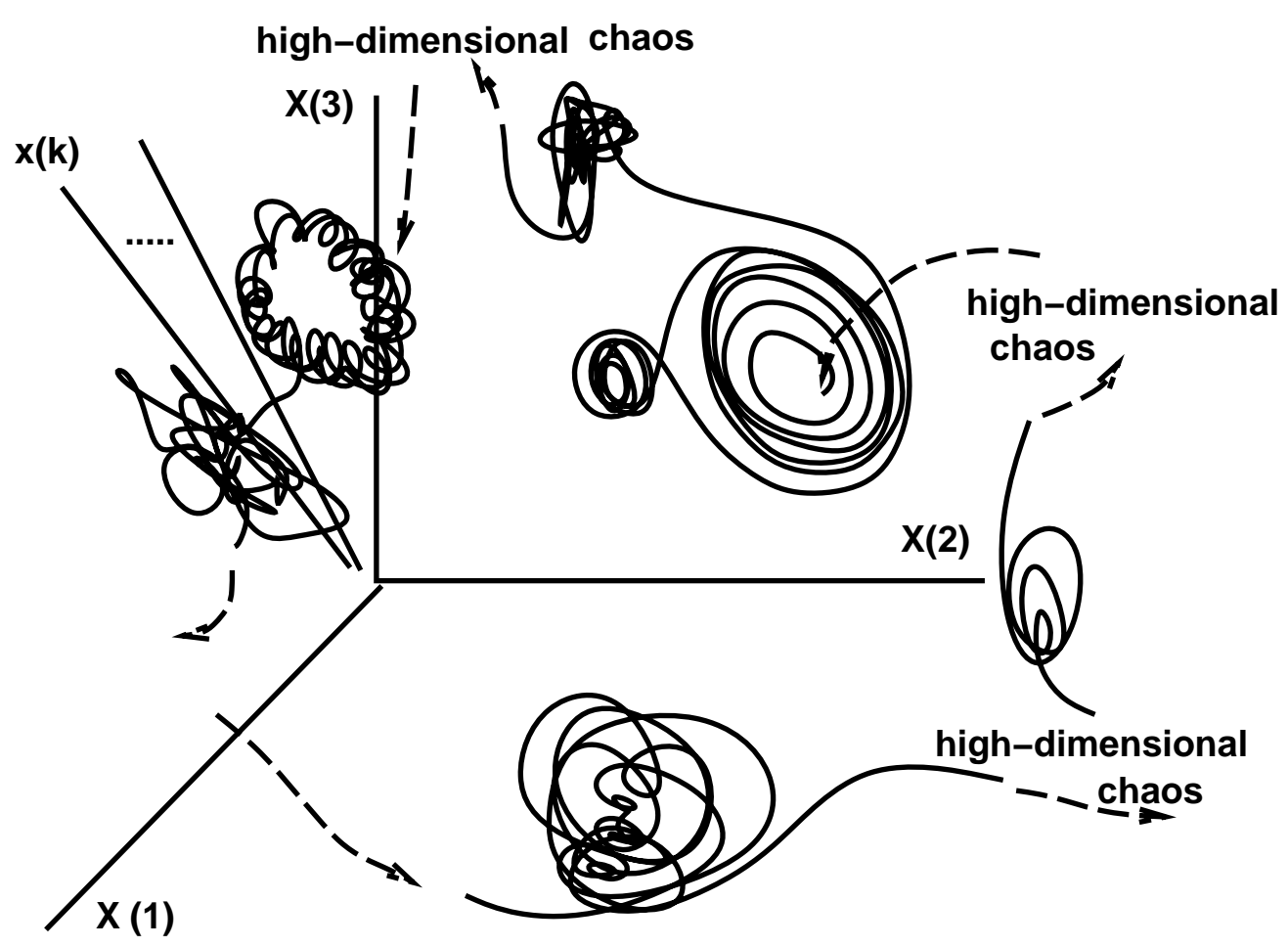

Figure 1: Schematic representation of chaotic itinerancy

phase space. Such low-dimensional motion is not described by a stable attractor, even though orbits are attracted to its vicinity. After staying at an attractor-ruin, an orbit exits from it. This exit arises from a certain kind of instability.

With this instability the orbits enter into a high-dimensional chaotic motion, losing coherence or correlation among variables. This high-dimensional dynamic state is also quasi-stationary, although after this chaotic wandering the orbit is again attracted to one of the attractor ruins which again possesses low dimensionality. In other words, there are some 'holes' connecting to attractor-ruins from the high-dimensional chaotic state. Once the orbit is trapped at a hole, it is suddenly attracted to one of attractor ruins, i.e., low-dimensional ordered states.

Now, a mechanism of the above instability should be solved. In this focus 
issue, several possibilities for the mechanism are addressed.

\section{Characteristics of chaotic itinerancy}

The basic characteristics of CI are as follows:

\subsection{Existence of low-dimensional ordered motion (at- tractor ruin)}

To exhibit CI behavior, there are several invariant subsets that are in lowdimensional space in the phase space. These subsets work as attractor-ruins.

In high-dimensional phase space, there can be stable and unstable (invariant) manifolds connecting each of invariant sets. To realize attraction to the neighborhood of this invariant set, the dimension of stable manifold must not be negligible in a whole space, in other words, at least a Lebesgue measure of the basin of such an invariant set is positive. On the other hand, this low-dimensional manifold cannot be an attractor in the whole space since there is at least non-zero dimensional unstable manifold along which the orbits leave the neighborhood of such an invariant set. In this regard, it is natural to call the geometric structure representing such low-dimensional ordered motion an attractor ruin.

In dynamical systems having some symmetry, there exists the case that a whole system can be decomposed into several low-dimensional invariant subspaces, according to which the whole dynamical behavior can be characterized by the union of the behaviors in subspaces. As is clearly seen by the definition of invariant subspace, once the motion is restricted to this low-dimensional subspace, the orbit stays there.

As a typical example, let us consider a globally coupled system consisting of identical elements showing chaotic dynamics (see $\S 3$ for details). Synchronization of all elements provides a chaotic state. This chaotic state is invariant under any permutation of elementary maps. Since this group action commutes with the dynamical system, this chaotic state is also invariant under temporal evolution. Furthermore, there could be states obtained by partial synchronization, i.e., synchronization only over some elements. Then, each of the states constitutes an invariant subspace. Some of these state can have positive Lebesgue measure of the basin of attraction. If this invariant set is unstable in a transversal direction to its invariant subspace, then the 
orbit is directed to other invariant subspaces from such chaotic invariant set. In such a case, wandering dynamics is observed as CI, and each invariant set, i.e., attractor restricted to each invariant space, is an attractor ruin.

\subsection{Escape from low-dimensional motion through re- stricted path}

After the escape from low-dimensional states, the motion becomes highdimensional. Since the region that an orbit escaping from an attractor ruin wanders is restricted in spite of this high-dimensionality, the itinerancy from one attractor ruin to another is distinct from random hopping among attractors with a help of external perturbations. On the other hand, due to this restricted path, the system maintains memory concerning the motion on attractor ruins which the orbit previously visited, and hence the historydependent orbits are generated.

As mentioned above, dynamics for an exit from an attractor ruin is not represented by simple noise motion. An aspect of chaotic motion for the escape from an attractor ruin, however, may be expressed by state-dependent noisy dynamics. Then, it may be possible to discuss this aspect, by using a state-dependent noise, as a first-step approximate description. For example, escape statistics from an ordered state may be discussed by using multiplicative noise, where the noise strength explicitly depends on the state. In the present issue, Nakao and Mikhailov discusses this aspect.

\subsection{Importance of marginal mode}

In CI, the attraction to low-dimensional motion and the escape from it are somewhat balanced. There are two extreme cases. If the former tendency wins, the motion falls onto a low-dimensional attractor, while if the latter wins, a high-dimensional irregular motion appears. For CI, balance between the two tendencies is required.

This balance leads to neutral stability of some modes. The stability of this kind can be described by the Lyapunov spectra. As far as we have verified in numerical simulations of several high-dimensional dynamical systems exhibiting CI, there appear many exponents whose values are close to

zero. The accumulation of the Lyapunov spectra to null exponents is one characteristic feature common to CI we have studied so far (see e.g., [9]). 
This characteristic leads to a new type of instability that Sauer studies in this focus issue. He focuses on the study of a two-dimensional map with extremely slow convergence or non-convergence of zero-Lyapunov exponent and its large fluctuation. Although chaotic itinerancy is a concept in high-dimensional dynamical systems, Sauer's model expresses one of essential characteristics of chaotic itinerancy. Sauer investigates the possibility of sensitive dependence on additive noise to this skeleton model and found the scaling law for natural measure. This suggests that the break of global stability is one of essential characteristics of chaotic itinerancy.

In CI large fluctuations of null Lyapunov exponents are expected, as Sauer treats in his article. Indeed, fluctuations in the largest Lyapunov exponents are addressed as a typical case of CI in this focus issue by Tsuda and Umemura.

\section{An explanation of chaotic itinerancy in a symmetric dynamical system: A globally coupled map}

One of the simplest models for high-dimensional dynamical systems is globally coupled dynamical systems. In particular, "globally coupled map" (GCM) consisting of chaotic elements [4] has been extensively studied, as a simple prototype model for chaotic itinerancy. A standard model for such GCM is given by

$$
x_{n+1}(i)=(1-\epsilon) f\left(x_{n}(i)\right)+\frac{\epsilon}{N} \sum_{j=1}^{N} f\left(x_{n}(j)\right)
$$

where $n$ is a discrete time step and $i$ is the index of an element $(i=1,2, \cdots, N$ $=$ system size). The map $f(x)$ is chosen so that the dynamics $x_{n+1}=f\left(x_{n}\right)$ shows chaos. In particular, the logistic map $f(x)=1-a x^{2}$ is often adopted. The model is just a mean-field-theory-type extension of coupled map lattices (CML) [8].

Through the average interaction, elements tend to oscillate synchronously, while orbital instability leads to destruction of such coherence. In the former limit, all elements oscillate coherently (Coherent phase), while elements are completely desynchronized in the other limit of strong orbital instability 
(Desynchronized phase). Between these cases, there is a case that elements split into clusters in which they oscillate coherently. Here a cluster is defined as a set of elements in which $x(i)=x(j)$. Attractors in GCM are classified by the number of synchronized clusters $k$ and the number of elements for each cluster $N_{i}$. When the parameter characterizing nonlinearity (e.g., $a$ in the above logistic map case) is not large, observed attractors have a small number of clusters $k$. If the number of cluster $k$ is small, the corresponding orbit is attracted to just low-dimensional attractor, i.e., at most $k$-dimensional. As the parameter is increased, these low-dimensional states lose the stability. If the instability is not large enough to lead to complete de-synchronization, the system often shows chaotic itinerancy. Here, lowdimensional attractor-ruins are given by a state with few number of clusters.

As an example, consider the case that this attractor ruin is given by a two-cluster state. Then the system is attracted to neighborhood of twodimensional plane $\left(x_{1}, x_{2}\right)$ in the $N$-dimensional phase space. In CI, after the orbit stays in the neighborhood of this two-dimensional plane it leaves out of the plane. During the exit process, often the orbit approaches almost coherent state, i.e., the state with $x_{1} \approx x_{2}$. In this GCM, when elements are totally synchronized, the dynamics are approximately represented by just a single map $x_{n+1}=f\left(x_{n}\right)$. Here this map shows stronger orbital instability than a clustered state with a few clusters. Hence as the orbit approaches this one-dimensional subspace in the two-dimensional subspace, it exists from the original two-dimensional subspace. ( see also schematic Fig.2). It is interesting to note that the effective degrees of freedom decreases before high-dimensional chaotic state appears. An explicit experimental realization of globally coupled dynamical systems is given by Kiss and Hudson in the present issue, as will be described later.

Let us reconsider basic characteristics in CI in this example. First, in this globally coupled dynamical system, invariant subspace is clearly defined from symmetry, whereby one can define a low-dimensional attractor ruin. On the other hand, high-dimensional chaotic motion is given by desynchronized dynamics. Second, paths to go out from the attractor ruin are given by orbits passing through further restricted subspace among the subspace for the attractor ruin. Third, when CI is observed, de-synchronization and synchronization are balanced over long term. This leads to the accumulation of null Lyapunov exponents, as discussed in [9].

As discussed above, each attractor-ruin in GCM is given by clustered state, characterized by the number of elements in each cluster $\left(N_{1}, N_{2}, N_{3}, \ldots, N_{k}\right)$. 


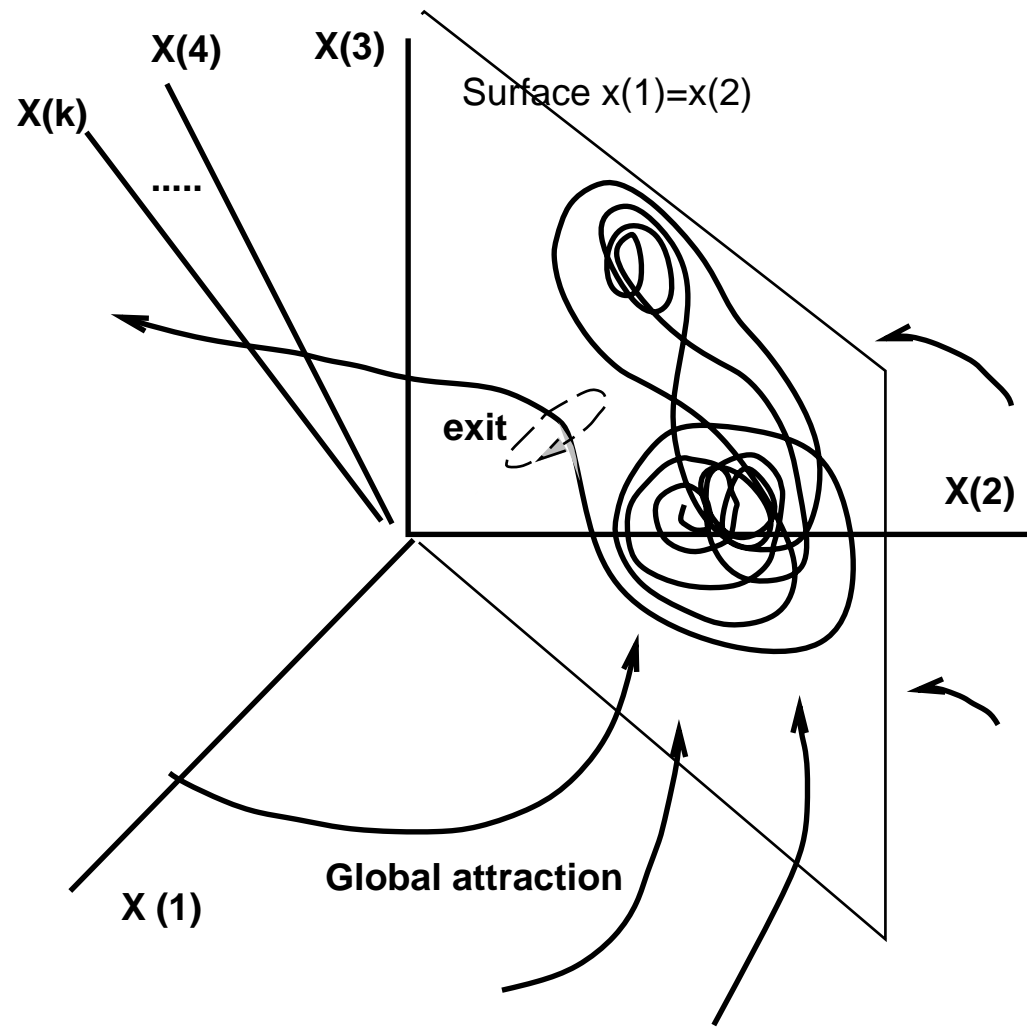

Figure 2: Schematic representation of attraction to, and escape from, an attractor ruin in chaotic itinerancy

Hence stability analysis of clustered states gives a basis for the study of CI in GCM. Maistrenko and Panchuk analyzed the stability of clustering solution, as well as its basin in detail. In particular, they analyzed basin structure of partly desynchronized attractor, i.e., clustering with $\left(N_{1}, 1,1, \ldots, 1\right)$ with $N_{1} \gg 1$.

To study attractor ruins, we need to deepen our understanding in longlasting transients within high-dimensional dynamical system. By using onebody distribution of the each value $x$ for each element also, Chawanya analyzed existence of such quasi-stable state and the transient length in a GCM consisting of tent maps. 


\section{Milnor attractor as a possible representa- tion of attractor ruin}

An attractor ruin in CI possesses structure of both global attraction to it from high-dimensional manifolds and escape from it through restricted region of its neighborhood. This structural feature is similar to that of a Milnor attractor. In this section, we discuss possibility to characterize an attractor ruin with a Milnor attractor[10, 11].

Milnor attractor is defined as a (minimal) set that has positive measure of its basin of attraction [12]. This definition does not exclude the possibility that the orbits leave from the attractor, namely, the presence of unstable manifolds of the attractor. This is clearly excluded in the definition of conventional geometric attractor. Therefore, Milnor attractor is an extended concept of attractor and includes the conventional geometric attractor.

In this way, Milnor attractor possessing unstable manifolds is unstable by perturbations of arbitrarily small size, but it still globally attracts typical orbital points. Here, we use the term Milnor attractor, only if it does not belong to the geometric attractor. If this Milnor attractor is chaotic, the basin is considered to be riddled [14]. This is the case for the present GCM model.

Existence of Milnor attractor in a symmetrically coupled system was first noted by Pikovsky and Grassberger[7]. The authors discuss a coupled map of two identical elements. Still, the Milnor attractor is not so common in a coupled system with few elements. Indeed, since Milnor attractor in the above sense is not asymptotically stable, one might, at first sight, think that it is rather special, and appears only at a critical point like the crisis in the logistic map[12]. Often, such critical points exist only at specific points in the parameter space. Hence Milnor attractors might look non-generic.

However, to our surprise, Milnor attractors are rather commonly observed around the border between the ordered and partially ordered phases in a globally coupled map [10,11], when the number of degrees of freedom is larger than $5 \sim 10$. It is suggested that the Milnor attractors are prevalent when the degrees of freedom having instability, globally coupled each other, is larger than $5 \sim 10[17]$. This number $5 \sim 10$ is also termed as magic number $7 \pm 2$, borrowing the terminology in psychology[18]. We will come back to this problem at $\S 6$.

A Milnor attractor is invariant under the group action, say substitution of 
elementary individual systems, and is invariant under the dynamics because of commutability between the dynamics and the group action. Therefore, a Milnor attractor forms an invariant set and can be an attractor ruin. In this case, the presence of Milnor attractor is closely related to the appearance of riddled basin.

Note that the Milnor attractors satisfy the condition of the above ordered states constituting chaotic itinerancy. Some Milnor attractors that we have found maintain global attraction, which is consistent with the observation that the attraction to attractor ruins in CI is global starting from a highdimensional chaotic state. Here, note that attraction of an orbit to precisely a given attractor requires infinite time in differentiable dynamical systems, and therefore before the orbit is completely attracted to a given Milnor attractor, it may be kicked away. Then, the long-term dynamics can be constructed as the successive alternations of the attraction to, and the escape from, Milnor attractors. Hence the dynamics is represented by transition over Milnor attractors. This transition is generally asymmetric: when there is a connection from a Milnor attractor A to a Milnor attractor B, but not necessarily from B to $\mathrm{A}$. The total dynamics is represented by the motion over a network, given by a set of directed graphs over Milnor attractors. In general, the 'ordered states' in CI may not be exactly Milnor attractors but can be weakly destabilized states from Milnor attractors. Still, the attribution of CI to Milnor attractor network dynamics is expected to work as one ideal limit.

To make itinerancy over Milnor attractors recurrently, there should be multiple Milnor attractors in the system, and with time evolution, Milnor attractors should be visited repeatedly.

A system with multiple Milnor attractors is easily constructed by coupling dynamical systems, each of which produces a Milnor attractor. Using a CML constructed as such, Tsuda and Umemura investigated the role of Milnor attractor. They discovered that the CML with a system size 5, which is an intermediate case of CML and GCM in its effective coupling, produces CI via a "higher order" Milnor attractors, while a CML with a larger size produces, if any, only transient CI. Here, global coupling is necessary to produce CI via Milnor attractors, while the result also suggests the plausibility of the above magic number $7 \pm 2$ with respect to universal observation of Milnor attractors.

Existence of Milnor attractors in CI is not restricted to coupled map systems. In neural network model by Tsuda, the Milnor attractor is likely to exist. Indeed, the observation of 'critical circle map' in the collective activity 
of neurons in the model $[2,3]$ is a possible manifestation of it.

\section{Classification of CI in a general case}

\subsection{With regard to symmetry}

In the last section, we have discussed CI based on a study on a dynamical system with symmetry. In globally coupled dynamical system of identical elements, there is permutational symmetry, while in a lattice system (CML), there is translational symmetry. In general high-dimensional dynamical systems, such symmetry does not exist. Still, the study with the symmetric case may be relevant to a general case also.

Case I: With some transformation, the system may be mapped into a symmetric case. Let us consider a coupled dynamical system with nonidentical elements. In this case, with some nonlinear transformation of variables, discussion for the symmetric case will be applicable. Consider phase synchronization, for example. Elements are not completely synchronized, but after some transformation of variables, they are regarded to be synchronized. Such generalization will be relevant to CI, in particular, for a globally coupled dynamical system with heterogeneous elements, including a coupled dynamical system consisting of elements with different time scales.

The paper of Fujimoto and Kaneko studies CI in a coupled dynamical system with distributed time scales (see also [19] for CI with distinct time scales). The model consists of elements with the same dynamics except their time scale. It is mapped to identical elements with the transformation of time scale by each. This coupled chaotic system with multiple time scales exhibits chaotic itinerancy with a mechanism of bifurcation cascade. Fast dynamics successively change slow dynamics, so that correlation is transferred to elements with a huge time scale difference. The work by Fujimoto and Kaneko opens a way to study chaotic itinerancy in frequency space.

The above theoretical paper is also considered to give a model for dynamic effect of this intermingled time scales, studied by Kay for neural activity, as will be discussed in $\S 7.4$.

Case II: Intrinsically asymmetric case

Generally, invariant subspace is not directly defined by the symmetry. In a typical situation for generating CI in this case, invariant set possesses both stable and unstable manifolds (which are invariant) and the transition 
between invariant sets can occur via these invariant manifold.

\subsection{From hypercycle to chaotic itinerancy}

As a transition over low-dimensional invariant sets, heteroclinic cycles are common with CI, and may be important for another possible mechanism of CI. In the heteroclinic cycle, an orbit visits successively the neighborhoods of the saddle-type fixed points. Here an orbit approaches a fixed point from the direction of its stable manifold, and after approaching close neighborhood of it, the orbit goes out of it through its unstable manifold and then asymptotically approaches a stable manifold of another fixed point. In other words, one unstable manifold of a fixed point is a stable manifold of another fixed point, and these manifolds form a cycle to switch among fixed points. Existence of such heteroclinic cycles has been discussed most extensively in a class of population dynamics model[20].

However, in such a case if the heteroclinic orbits become chaotic or not is problematic. Heteroclinic cycles originally studied are non-chaotic and structurally unstable and are not suitable for a mathematical framework for chaotic itinerancy. It is possible to introduce chaotic dynamics to it as studied, for example, by Chawanya[21]. In the present issue, Ashwin et al. introduced a model for robust cycling between chaotic and equilibrium saddles. They explicitly design a system for robust cycle between invariant sets and saddles. In general, it will be important to study a robust cyclic process, by "thickening" connection paths among invariant sets, so that the motion can include high-dimensional chaotic dynamics. Such system gives one prototype of CI.

In general, saddle-type structures in phase space are important to chaotic itinerancy. In relation, the saddle-type structure in infinite dimensional systems is studied in this issue by Nishiura, Teramoto and Ueda. Chaotic itinerancy that appears in a dynamical system with continuous space and time is analyzed by the interactions of saddle-type structures in phase space. A specific saddle-type structures has been discovered and analyzed. They studied a transitory dynamics of particle-like patterns in Ginzburg-Landau equation, the Gray-Scott model and a three-component reaction diffusion model, and suggest that localized steady or time-periodic saddle-type structures, called scattors, may play an important role in chaotic itinerancy in many PDE systems.

Freeman studies in this focus issue human EEG with his 1x64-electrode 
system that is a nontrivial extension of $8 \times 8$-electrode system that he invented for the previous studies of animal olfactory bulb. Freeman has discovered a curious instability that reveals sudden jumps in phase which are synchronized in a very wide range. Such jumps are asynchronous across the midline separating the left and right hemispheres and also across the central sulcus separating the frontal and parietal lobes. The time duration between sudden jumps changes chaotically. This indicates that chaotic transition between synchronized states occurs in some areas of the cortex and also between asynchronous states in other areas. Since a transition is very sharp, this may be related to the transition by the appearance of heteroclinic cycle. If the existence of heteroclinic orbits is verified, the present findings of Freeman could provide a new example of CI.

\subsection{Classification by the form of ordered motion}

Each motion at an attractor ruin is not necessarily chaotic. In some case, it can be fixed point, limit cycle, or quasi-periodic. For example, CI observed in Otsuka et al.'s paper is based on quasiperiodic and frequency locking states. If the fixed point is ordered motion, noise is necessary to make a switching as is discussed in nonequilibrium neural network model by Tsuda[2].

The noise may play a role of producing positive measure of support of ordered motion which allow the riddled basin to appear. Kozma treats KIII model originally introduced by Freeman, and discusses a type of "attractor crowding". Kozma also found a similar noise effect to that Liljenstrom found in a model of the olfactory system. Chaotic itinerancy with multiplicative noise that was introduced in the form of stochastic renewal of neural dynamics was studied by Tsuda et al about fifteen years ago. Statistics of bursts from a stationary state by multiplicative noise is also studied by Nakao and Mikhailov.

A similar transition phenomenon in the system with riddled basins has also been found by Grebogi et al [15], by introducing additive noise to such a system. Kozma in the present article addresses the role of additive noise

in the "attractor crowding" of KIII model. He discovered a stabilization of chaotic orbits by noise and also the appearance of maximum in signal to noise ratio in the chaotically itinerant state. 


\subsection{Degree of path}

There seem to be several types of "chaotic itinerancy" covered by this general definition for it. One classification may be possible according to the degree of correlation between the ordered states visited successively. If the paths for the transitions between the ordered states are narrow, the correlation is high and the probabilities for visiting the next ordered state are rather low. On the other hand, the correlation is low if the memory on the previous sates is lost due to high-dimensional chaos during the transition.

\section{1) Cases with strong correlation:}

If the connectivity among degrees of freedom is sparse, the path can often be very much restricted to some specific portion in the total phase space. For example, consider a system with local coupling in space such as the one typically realized in coupled map lattice(CML). Switching over several traveling wave patterns is observed in this CML [16], where the traveling wave is a global phenomenon covering all lattice points. Hence, the switching of wave patterns can be interpreted as an example of chaotic itinerancy. In this case, although the transition occurs through a chaotic state, its dimension is too low to allow for a variety of destinations. Indeed, the transition over wave patterns is rather limited. It seems that CI in optical trubelnce by Ikeda[1] also belongs to this class.

The extreme case for low-dimensional path is heteroclinic cycle, where connections are made only by one-dimensional unstable and stable manifolds. Chaotic itinerancy realized by 'thickening heteroclinic cycle' as discussed in $\S 4.2$, has still limited path connection, and belongs to this case with strong correlation.

2) Cases with weak correlation:

For the chaotic itinerancy in the GCM, there is a huge number of invariant sets, given by the clustering condition. The chaotic itinerancy occurs among such a huge number of possible attractor ruins. If the orbit passes through high-dimensional desynchronized state, and it lasts relatively long, there remains little correlation between two successive attractor ruins. The memory of the previous state decays during the transition.

3) Cases with medium correlation:

In GCM, all elements are connected to all others, which make paths rather high-dimensional. By restricting the connection, the path has more restriction. For example, Nozawa studied a GCM where the coupling strength among the elements is not uniform but sparse, by borrowing Hopfield-type 
neural network[22]. As a result, the number of "attractor ruins" is reduced and the paths between such attractor ruins are much limited. Hence there is larger correlation between the patterns before and after a transition. Such restriction in the path is also imposed in Tsuda's nonequilibrium neural network model[2], which leads to long-time hysteresis over retrieved patterns.

In these chaotic itinerancy models, the unstable manifold of the attractor ruins has a relatively low dimension. In this respect, the correlation between memory states may determine the dimensionality of the transition in such a way that a strong correlation generates a low-dimensional transition path, whereas a high-dimensional transition path is generated in a weak correlation case.

\section{Ubiquity of CI in a "high"-dimensional sys- tem}

Through numerical studies for a variety of models with many degrees of freedom, chaotic itinerancy seems to be ubiquitous, when the system is neither too disordered losing correlation among degrees of freedom nor too ordered attracted to low-dimensional attractors.

One question here remains: How many dimensions are required to be "sufficiently high-dimensional"? How high should the connection among degrees of freedom be? Or, in other words, to what point of the argument so far, dimensionality or connectivity is relevant?

There is not decisive answer as yet, although relevance of combinatorial complexity is discussed in [17]: As the number of coupled elements $N$ increases, the combinatorial variety of grouping (clustering) of these degrees of freedom increase with the factorial order of $N$ (say $(N-1)$ !), while the volume of phase space expands only exponentially. Hence the combinatorial variety surpasses the increase of phase space volume at some $N$, beyond which the distance between an attractor and its basin boundary may drastically decrease, leading to the increase of fraction of Milnor attractors. Now, several attractor-ruins are formed, and CI may be rather commonly observed.

Let us elaborate the above discussion a little bit. Consider a one-dimensional phase space, and a basin boundary that separates the regions of $x(1)>x^{*}$ and $x(1)<x^{*}$, while the attractor in concern exists at around $x(1)=x_{A}<x^{*}$, and the neighboring one at around $x(1)=x_{B}>x^{*}$. Now consider a region 
of $N$-dimensional phase space $x_{A}<x(i)<x_{B}$. If the region is partitioned by (basin) boundaries at $x(i)=x^{*}$ for $i=1, \cdots N$, it is partitioned into $2^{N}$ units. Since this partition is just a direct product of the original partition by $x(1)=x^{*}$, the distance between each attractor and the basin boundary does not change with $N$.

On the other hand, consider a boundary given by some condition for $(x(1), \cdots, x(N))$, represented by a (possibly very complex) hyperplane $C(x(1), \cdots, x(N))=$ 0 . In the present system with global couplings, many of the permutational changes of $x(i)$ in the condition also give basin boundaries. Often, the condition for the basin can also have clustering $\left(N_{1}, \cdots, N_{k}\right)$, since the attractors are clustered as such. Then the condition obtained by the permutation of $C(x(1), \cdots, x(N))=0$ gives a basin boundary also. The number of such segments of the boundaries increases combinatorially with $N$, roughly speaking in the order of $(N-1)$ !, when a variety of clusterings is allowed for the boundary. Now the $N$-dimensional phase space region is partitioned by $O((N-1)$ !) basin boundary segments. Recalling that the distance between an attractor and the basin boundary remains at the same order for the partition of the order of $2^{N}$, the distance should be drastically decreased if $(N-1)$ ! surpasses $2^{N}$. Since for $N>5$, the former increases drastically faster than the latter, the distance should decrease drastically for $N>5$. Then for $N>5$, the probability that a basin boundary touches with an attractor itself will be increased. Since this argument is applied for any attractors and their basin boundary characterized by complex clusterings having combinatorial complexity. Although this explanation may be rather rough, it gives a hint to why Milnor attractors are so dominant for $N \gtrsim(5 \sim 10)$. In this sense, the dimension necessary for prevalence of CI could be "magic number seven plus minus 2" [17].

As discussed above, attractors are sometimes crowded in the phase space if the dimension of the phase space is "high". Freeman and his colleagues discuss this attractor crowding, in a model of the olfactory bulb, so called KIII model. A mesoscopic system in each different hierarchy is studied by Freeman, Kozma and Kay also in this issue. 


\section{$7 \quad$ Chaotic Itinerancy in nature}

\subsection{Chaotic itinerancy in Hamiltonian system:relevance to energy conversion}

So far we have discussed CI in dissipative system, where attractor concepts are important. To a Hamiltonian system, the attractor concept is not applicable. Still, itinerant motion over several quasi-stationary states is often observed in a Hamiltonian system with many degrees of freedom. There, the system in concern is confined at a state with some ordered structure over long time span, while chaotic motion with a small amplitude remains. At this ordered structure, degrees of freedom are separated into groups, and elements (modes) within each group show highly correlated motion. On the other hand, motions of elements belonging to different groups are approximately disjointed. After residence at one of such ordered states, degrees of freedoms that were roughly disjointed start to interact, which makes the system leave out of the ordered state. There, with mode couplings, stronger chaotic motion appears, which later is replaced by another ordered state suppressing strong chaos and separation of degrees of freedom. In this sense, it is fair to adopt the term chaotic itinerancy for such Hamiltonian dynamics also.

This type of CI was first discussed by Konishi and Kaneko[23], in a globally coupled pendulum system, while similar dynamics were soon observed in self-graviton system[27].

In some sense, one might think that such itinerancy over several ordered states is just thermal hopping dynamics over energy local minima in a potential landscape, as was traditionally discussed. Although both the CI dynamics here and the standard hopping process are common as transition phenomena over states, the transition in CI is clearly distinguishable from a thermal activation process. CI is due to deterministic dynamics with several degrees of freedom, and not by random motion from heat bath. In the itinerant motion, the energy is not dissipated to whole degrees of freedom as "heat". This feature may bring about deviation from traditional Arrhenius law, i.e., the transition probability with $\exp (-\Delta E / k T)$ form for the switching from one state to another.

Hence, chaotic itinerancy may give a new insight to problems so far discussed just as random hopping among several metastable states over energy barrier. In contrast to this standard viewpoint, there can be a directional motion for the switching from one state to another in CI. Itinerant motion 
in molecular dynamics of glass is studied by Shinjo[24] from this viewpoint, while molecular dynamics simulation of water again suggests non-random itinerancy over several inherent structures[25]. Recently, dynamics of surface atoms penetrating into micro-clusters is investigated by Kobayashi et al.[26], where rapid diffusion of atoms is observed as a universal feature of small clusters, and is discussed from a viewpoint of chaotic itinerancy.

Macromolecules like protein may have complex dynamics within, and are also candidates for a system to exhibit CI. Indeed, protein folding was discussed from this viewpoint by Matsumoto, while a simpler model for it is included in this issue by him. He has found unidirectional motions with high probability in the folding process, in a Hamiltonian system of doublewell applied random perturbations. He discovered a dynamical mechanism of the appearance of this non-ergodic motion.

Directional motion in CI may also give a robust mechanism for energy conversion. This problem is discussed by Nakagawa and Kaneko in the present issue, who describe conversion of injected energy to a certain directed motion in a model inspired by molecular motor experiments studied as an elementary process of muscle contraction. They simulate a simple Langevin dynamics with several degrees of freedom and have found relevance of transient chaotic behavior to energy conversion.

Both of the two systems are dynamic mesoscopic system in the sense that a macroscopic state cannot be represented by a simple order parameter but a certain time-dependent ordered motion at a mesoscopic level is important. These models suggest that CI plays an essential role in the energy transfer of macromolecular systems by activating the process of conversion from ergodic motion to non-ergodic motion. In general, it will be important to study functions of enzyme or molecular motor from the viewpoint of CI.

\subsection{Chaotic itinerancy in physico-chemical experiments}

A variety of physical and chemical systems with many degrees of freedom shows itinerant motion over several quasi-stable ordered states, through irregular motions associated with many degrees of freedom. So far, in the traditional picture taken for such phenomena, one assumes each state as a "metastable state" as in the local minimum of potential landscape, and then assume the switching over the states as random hopping over barriers triggered by (thermal or other) noise. However, in recent observations, there are several phenomena that do not fit well with such stan- 
dard "static"+"stochastic" picture. These phenomena may be understood as chaotic itinerancy.

An example in physics was first presented in optics with excitations of multiple modes. Indeed, one of the first discoveries of chaotic itinerancy was due to theoretical model of optical turbulence, as mentioned. Later, further examples are found both theoretically and experimentally[28, 29, 30]. In this present issue, Otsuka et al. studied dynamics of globally-coupled three-mode laser, and observed self-induced switching over several modes of oscillations.

Global coupling often lead to itinerant dynamics. An example is given by Nasuno's experiment on quasi-two-dimensional gas-discharge system. In the system, localized, luminous spots are formed. With the increase of discharge current, they form a 'molecular-like cluster', which, with further increase of the current show switching over several quasi-stable internal structures, with intermittent rearrangement of mutual positions of spots. Whether this switching process is understood as chaotic itinerancy or not is yet open, but this itinerancy, at least, seems rather different from random hopping over the local structures.

One of the most beautiful experimental demonstrations of globally coupled chaotic systems is an array of coupled electro-chemical oscillators, developed by Kiss and Hudson[31, 32]. Previously they showed that the system shows coherent, ordered, and desynchronized phases, with the change of coupling parameter among arrays, where clusters of elements with mutual coherent oscillations are formed. In the paper in the present issue, they showed experimental demonstration of chaotic itinerancy, by measuring the precision-dependent clusters and analyzing them as hierarchical cluster trees, as introduced in [4]. Change of effective degrees of freedom is directly computed. Their results show remarkable similarity with those observed in globally coupled maps.

As fluid turbulence provided a variety of examples for chaos, it can also be an experimental testbed for chaotic itinerancy. In a coupled map lattice model for Rayleigh-Benard convection, switching over several roll patterns is observed and characterized as chaotic itinerancy[33]. There are also corresponding experimental results, although detailed analysis as chaotic itinerancy is not yet published. In electrohydrodynamic convection of nematic liquid crystal, Sano et al. [34] observed formation and collapse of target patterns, that may belong to chaotic itinerancy over several patterns also.

At a much larger scale, atmosphere dynamics may exhibit chaotic itin- 
erancy. Indeed, by using an atmospheric model with realistic topography, Itoh and Kimoto[35, 36] found a CI phenomenon, in which the atmosphere system stays within attractor ruins for a long time, moving promptly among them. They found preferred routes in transitions among attractor ruins. Interestingly, these characteristics coincide well with those of weather regimes in the real atmosphere.

\subsection{Chaotic itinerancy in biology}

Biological system is often composed of many dynamic elements. A multicellular organism consists of cells with internal biochemical reaction, while an ecosystem consists of populations of several species. Due to dynamics of elements, they sometimes synchronize, or form a cluster of synchronized elements, or differentiate into several clusters. Sometimes, these dynamics are not stationary, but the clusters may change in time. There CI may appear, and can be relevant to dynamics of mutual relationship, to provide evolvability, and to sustain diversity[6, 37]. Although decisive experimental demonstrations are not yet available, several theoretical models are studied, to imply possible relevance of chaotic itinerancy for evolvability of biochemical reaction dynamics[38], differentiation from stem cells[39], and diversity in an ecological system[46] (see also $§ 8.2$ ).

\subsection{Brain}

Chaotic itinerancy has also been addressed in neurophysiological experiments with animals and even human. Freeman established the presence of chaotic behavior, its realization by noise through attractor crowding and its functional significance. The transition between multiple wings of quasi-attractors during a motivated perception process and also during learning was interpreted by chaotic itinerancy. Kay observed an itinerant behavior in local field potential as characterized to be non-stationary transitory dynamics in the animal cognitive and behavioral experiments with rats brain including an entire olfactory system and hippocampus. Recently, Freeman discovered a curious transition phenomenon in human EEG. He tries to interpret, in this issue, the sharp transition in terms of chaotic itinerancy, as we already mentioned. Kay found a dynamics at three time scales: slow, fast, and intermediate modes. Among others, medium time scale events unpredictably change with a strong correlation with animal performance. Kay proposes 
in the present issue the folding of different time scales as a mechanism for chaotic itinerancy.

Another important finding in physiological experiments in relation with chaotic itinerancy is chaotic alteration between synchronization and de-synchronization of the activity of neural assemblies. The first finding was made by Gray [40]. He observed this type of chaotic alteration in cat visual cortex. It is often observed that integrated electric potential of neuron assemblies strongly correlates and is synchronized between even far separated regions when an appropriate stimulus is input. However, this synchronization does not last for a long time. It changes chaotically to desynchronized chaotic states after a few hundred milliseconds. This alteration looks like chaotic itinerancy. Tsuda, Fujii, Tadokoro and Yasuoka [41] have proposed a neuronal model with gap junction couplings that is a diffusion type of couplings to provide a dynamical mechanism of this chaotic alteration.

Raffone and von Leeuwen found an alteration between synchronization and de-synchronization in a layered network model with three-variable Hindmarsh and Rose model. They correctly discuss in the present issue the importance of chaotic transitory process in simultaneous retrieval of memories, taking into account cognitive behaviors. Although their synchronization and de-synchronization observed in assemblies of model neurons are relative, this seems to represent a rather realistic model.

Han and Postnov found chaotic itinerancy in the diffusively coupled Morris-Lecar neural system, where the transitory phenomena appears among unstable three states: in-phase and anti-phase synchronized states and small amplitude non-firing state. By their work in the present issue, it turns out that chaotic itinerancy provides a new route to chaos via resonant torus breakdown. The diffusive coupling adopted by Han and Postnov can be interpreted as a representation of gap junction coupling of neurons which has widely been observed in animal and human brain, even in the neocortex. Their system is relatively low-dimensional, but show a new mechanism of chaotic itinerancy which may hold also in high-dimensional systems like gap junction coupled neural systems.

A neural model for chaotic itinerancy is addressed in the present issue in two directions. One is a numerical study in a large scale system that is presented by Nara, and the other is a mathematical study in a prototype model of chaotic neural network of a small scale that is presented by Kitajima, Yoshinaga, Aihara and Kawakami.

Nara has studied an effect of chaotic search in the problem of association 
of memories. Nara and his colleagues have used a recurrent neural network with limit cycle oscillations which represent memories. In the present article, Nara clarifies that chaotic itinerant behaviors can exhibit high performance of memory search, compared with so called random search, and the performance can be better by learning because of the change of degree of constraint to access to each memory pattern even when chaotic search was worse than random search.

Kitajima, Yoshinaga, Aihara and Kawakami study one of possible mechanisms of chaotic itinerancy observed earlier by Aihara et al in a network of chaotic neurons. Kitajima et al treat in this article four coupled chaotic neurons with two orthogonal patterns which are stored in the network as a representation of memory. The model was reduced six-dimensional chaotic map which possesses three-dimensional chaotic subsystem. They analyzed the model and found the presence of the intersection of unstable manifolds of periodic points and collapse of in-phase-locked chaos as an essential process of the presence of chaotic itinerancy appeared in the model.

\subsection{Chaotic itinerancy at a psychological and a social system?}

Aiming at a higher behavioral or psychological level, Ikegami and Morimoto studied a coupled dynamical recognizer model. The study is inspired by the dynamics of turn-taking in conversation. The model is a coupled dynamical system, called coupled dynamical recognizer. Here, each dynamical system is a recurrent neural network, to play a game mutually. The state of neural network represents "image" on the strategy of other players. Ikegami and Morimoto used this framework for a three-person game in which coalition pair may be formed or change in time[43]. Due to the instability in learning dynamics, switching of the coalition pair is found, and is studied as chaotic itinerancy. Although there may be some gap between the model and

the psychological experiment at the present stage, it will be promising to discuss human psychological process (such as mutual understanding process) from the viewpoint of CI.

Itinerancy over several quasi-stationary states is often observed in social system. In human history, there are quiescent regimes interspersed by the regime of drastic change, to which the term 'revolution' is often assigned. Often, in this transition, order in the ancient regime is replaced by dynamic 
change of social structure, before novel order is formed. Although it may be too naive at the preset status of research to discuss social dynamics from the viewpoint of CI, it may provide a novel standpoint to analyze the social change.

In the study of history, most studies focus on estimating a chain of causal relationships so far. Chaotic itinerancy may give a framework to understand a mechanism for the change of relationships among elements, for generation and collapse of ordered structure, and for the dynamics of transition among ordered states. By choosing a problem concerning economics, in particular money, Yasutomi discusses the emergence and collapse of money from dynamical systems of many agents exchanging goods, in this issue.

\section{Significance of CI}

\subsection{Dynamics of relationship}

In a biological system, often several elements show correlated motion or work together. Activities of neurons are often correlated, while a group of genes is sometimes expressed together. In such case, often it is assumed that couplings are prepared to be strong within these elements forming correlated motion. Accordingly, existence of pre-defined 'module' is often assumed in neuroscience and in cellular biology.

Still, examples are increasing to suggest that these groups are not predefined, but can change in time. Groups of neurons or genes that work correlatively often change in time or change depending on external condition.

CI provides a novel viewpoint in such 'dynamic change of relationships' (see also, for example, [41]). In CI, groups of correlated action are formed at each attractor-ruin, as a result of dynamics, even without pre-defined strong couplings among the elements. The connection strength among elements in each group are not necessarily strong. The connection among elements may give restriction for the transition among attractor-ruins, but groupings are not necessarily determined by them.

In this sense, CI gives a new insight on how module-type structures are formed spontaneously, and also on how rules on dynamic change among these structures are generated.

As for a theoretical tool to understand generation of ordered motion with low-dimensional degrees of freedom, "dissipative structure" [44] or slaving 
principle [45] is often adopted. Chaotic itinerancy gives both formation of ordered structure and also its collapse, and furthermore generation of flexible rule, for this itinerancy. Here it should be noted that the itinerancy is a property of each element dynamics, but occurs at a macroscopic level defined by collective motion given by elements. In this sense, the rule for itinerancy is with regards to a higher level than each element dynamics.

\subsection{Evolvability and stability}

Biological system consists of a huge number of elements, say chemical species for a cell, species for ecosystem. How such high-dimensional dynamical systems keep stability is one of the key questions in a biological system. Furthermore, a biological system, although it is stationary over some time span, can also change in a longer time span, as are common in developmental dynamics and in evolution. Biological system satisfies both recursiveness to maintain its macroscopic state and changeability to a novel state as evolution. Here, the time regimes for recursiveness and evolution are sometimes separated, as seen in metamorphosis in development, and as discussed as punctuated equilibrium for evolution. Chaotic itinerancy gives a theoretical mechanism to stability for a recursive state and also to evolution. Stability of ecosystem is discussed with population dynamics of a variety of species [46] in relationship with CI, while recursive production of biochemical states and their evolution are studied with itinerant dynamics[38].

\subsection{Energy conversion}

As discussed in $§ 7.1$, CI may provide a robust mechanism for energy conversion within fluctuating environment. If the conversion occurs through "heat', mechanical energy given by a few degrees of freedom is once absorbed into many degrees of freedom. CI also gives switching between low- and highdimensional dynamics. In CI, however, itinerancy occurs through restricted paths within the total phase space. Hence the conversion may occur more robustly and efficiently within a limited time span. Deviation from Arrhenius law for crossing energy barrier discussed by Nakagawa and Kaneko may suggest such relevance of CI dynamics to energy conversion. 


\subsection{Searching process}

Searching process within high-dimensional phase space is often important in optimization and information processing. Since orbits in CI take only restricted regions in the phase space, search process with CI may be more efficient than random searching process, if the dynamics in the phase space are chosen properly. An example for such application was discussed by Nozawa[22], as a use of CI for traveling salesman problem.

\section{5 information processing}

Since in chaotic itinerancy "neutral" stable modes are immanent, which are realized in the successive transitions between ordered modes via chaotic motion. Therefore, the decay of the autocorrelations and mutual information due to chaotic motion is very slow; usually in power decays. In such a case, according to Matsumoto and Tsuda [47, 48, 49], an information mixing appears, thereby the information input to a certain element of the network could propagate to other elements before decaying. By the information mixing property, the information possesses a similar structure to hologram. In other words, each digit contains the content of whole information, though there is a quantitative difference. By this property, the input information is maintained within the network. The difference from the hologram is the way of maintenance of information: in a static manner in hologram, but in a dynamic one in chaotic itinerancy. Indeed, such global information cascade in CI is studied by using a bit space, for a GCM[9].

Furthermore, another significance of chaotic itinerancy in information processing lies in the use of temporal development. As Raffone and van Leeuwen show in the present issue, and Tsuda et al [50] and Nicolis and Tsuda [51] also show elsewhere, the motion of chaotic itinerancy could realize the multiplexing time series. This is particularly important when we consider a pattern association with a help of associative memory. When some patterns pop up in our mind, this must not kill other memorized patterns. When such a pattern disappears from our mind, another pattern should pop up for information processing. It turns out by the present work by Raffone and van Leeuwen that this is realized by neural networks exhibiting chaotic itinerancy that is chaotic alternation between synchronization and de-synchronization even in learning process.

Generation of various time scales in chaotic dynamics may allow for infor- 
mation propagation in modes with different time scales. CI, through correlations of ordered modes and chaotic dynamics may lead to such information propagation to modes with far distant time scales. Indeed, in biological system, modes with different time scales are intermingled. Furthermore, in "memory", fast time-scale changes are embedded into much slower modes. Papers by Fujimoto and Kaneko and Kay discuss this issue.

Significance of chaotic itinerancy in brain and cognition has widely been discussed by Tsuda and colleagues [42]. Among others, related to this issue, the simultaneous processing of learning and retrieval of memories is highlighted. In the appearance of chaotic itinerancy, the system which exhibits chaotic itinerancy can retrieve memorized patterns while learning. In usual neural network models, this simultaneity has been difficult to be realized, because without chaotic itinerancy only currently retrieved memory is object to be learned and such excess learning avoids the renewal of retrieval of memories.

Freeman [52] addressed a perceptual drift as a possible contribution of chaotic itinerancy to the level of perception and cognition. Perceptual drift is necessary particularly for pattern recognition and classification that we naturally experience in our daily life. This is a category formation in an extensive sense. For category formation, both identification of different patterns by finding similarity and differentiation of similar patterns by finding difference [41] are prerequisite. In order to make these processes compatible, perceptual drift is used to work at the cognitive level. Freeman's finding addressed in the present issue is a new evidence of perceptual drift. Since perceptual drift is deeply related to the way of formation of memories, especially episodic memories, the propagation and non-stationary transition of the activity of entire olfactory system and hippocampal formation with multiple time scales described by Kay may give a dynamical mechanism of perceptual drift underlying the formation of memories.

\section{References}

[1] K. Ikeda, K. Matsumoto, and K. Otsuka, "Maxwell-Bloch Turbulence" Prog. Theor. Phys. Suppl. 99 (1989)295

[2] I.Tsuda, in Microcomputers and Attention(Manchester University Press, 1991)430; World Futures 32(1991)167"Chaotic itinerancy as 
a dynamical basis of Hermeneutics in brain and mind" , World Futures 32(1991b)167.

[3] I.Tsuda, "Dynamic link of memory-chaotic memory map in non equilibrium neural networks", Neural Networks 5(1992a)313.

[4] K. Kaneko "Clustering, Coding, Switching, Hierarchical Ordering, and Control in Network of Chaotic Elements", Physica 41 D (1990) 137-172

[5] K. Kaneko “ Globally Coupled Circle Maps", Physica 54 D (1991) 5-19

[6] K. Kaneko and I. Tsuda Complex Systems: Chaos and Beyond $-A$ Constructive Approach with Applications in Life Sciences (Springer, 2000)

[7] A.S. Pikovsky and P. Grassberger " Symmetry breaking bifurcation for coupled chaotic attractors J. Phys. A 24 (1991) 4587-4597

[8] K.Kaneko ed., Theory and applications of coupled map lattices Wiley (1993); K. Kaneko ed., CHAOS focus issue on coupled map lattices, Chaos 2, 279-407 (1992).

[9] K. Kaneko, “ Information Cascade with Marginal Stability in Network of Chaotic Elements", Physica D 77 (1994), 456-472

[10] K. Kaneko, "Dominance of Milnor Attractors and Noise-induced Selection in a Multi-attractor System", Phys. Rev. Lett., 78 (1997) 27362739

[11] K. Kaneko, "On the Strength of Attractors in a High-dimensional System: Milnor Attractor Network, Robust Global Attraction, and Noiseinduced Selection", Physica D, 124 (1998) 322-344

[12] J. Milnor, "On the concept of attractor", Comm. Math. Phys. 99 (1985) 177; "Correction",ibid 102 (1985) 517

[13] P. Ashwin, J. Buescu, and I. Stuart, "BUBBLING OF ATTRACTORS AND SYNCHRONIZATION OF CHAOTIC OSCILLATORS" Phys. Lett. A 193 (1994) 126; "From attractor to chaotic saddle: A tale of transverse instability", Nonlinearity 9 (1996) 703 
[14] J.C. Sommerer and E. Ott., “ A PHYSICAL SYSTEM WITH QUALITATIVELY UNCERTAIN DYNAMICS", Nature 365 (1993) 138; E. Ott et al., "SCALING BEHAVIOR OF CHAOTIC SYSTEMS WITH RIDDLED BASINS", Phys. Rev. Lett. 71 (1993) 4134

[15] Kraut S, Feudel U, Grebogi C Preference of attractors in noisy multistable systems PHYS REV E 59: 5253-5260; 1999

[16] K. Kaneko "Global Traveling Wave Triggered by Local Phase Slips", Phys. Rev. Lett. 69 (1992) 905-908

[17] K. Kaneko , Dominance of Milnor attractors in Globally Coupled Dynamical Systems with more than $7+-2$ degrees of freedom Phys. Rev. E.66, 055201(R) 2002

[18] G.A. Miller, The psychology of communication, 1975, Basic Books, N.Y.

[19] H.Okuda and I.Tsuda, 'A coupled chaotic system with different time scales: Toward the implication of observation with dynamical systems', Intl. J. of Bifurcation and Chaos,4(1994)1011-1022.

[20] J. Hofbauer and K. Sigmund, Evolutionary Games ad Population Dynamics, Cambridge Univ. Press, 1998

[21] T. Chawanya, "Coexistence of infinitely many attractors in a simple flow", PHYSICA D 109 201-241 1997; "Infinitely many attractors in game dynamics system", Prog. Theor. Phys. 95 679-684 1996

[22] H. Nozawa, "Solution of the optimization problem using the neuralnetwork model as a globally coupled map", Physica D 75: 179-189 1994

[23] T. Konishi and K. Kaneko "Clustered Motion in Symplectic Coupled Map Systems", J. Phys. A, 25 (1992) 6283-6296

[24] Shinjo, K., "Formation of a glassy solid by computer-simulation", Phys. Rev. B40 (1989) 9167.

[25] Ohmine, I. and H. Tanaka, "Potential-energy surfaces for water dynamics. 2. vibrational-mode excitations, mixing, and relaxations", $J$. Chem. Phys. 93 (1990) 8138. 
[26] T R. Kobayashi, K. S. Ikeda Y Shimizu, S-i Sawada, "Isothermal dynamics simulations of spontaneous alloying in a microcluster", Phys. Rev. B 66, 245412 (2002)

[27] Tsuchiya T, Gouda N, Konishi T, "Chaotic itinerancy and thermalization in a one-dimensional self-gravitating system", Astrophys. and Space Science 257: 319-341 1998

[28] Otsuka K., "SELF-INDUCED PHASE TURBULENCE AND CHAOTIC ITINERANCY IN COUPLED LASER SYSTEMS" Phys. Rev. Lett. 65: 329-332 1990

[29] Arrechi F.T., Giacomelli G., Ramazza P.L, Residori S. "Experimentalevidence of chaotic itinerancy and spatiotemporal chaos in optics" Phys. Rev. Lett. 65: 2531-2534, 1990

[30] Fischer I, vanTartwijk GHM, Levine AM, Elsasser W, Gobel E, Lenstra D, "Fast pulsing and chaotic itinerancy with a drift in the coherence collapse of semiconductor lasers", Phys. Rev. Lett. 76: 220-223 1996

[31] W.Wang, I.Z. Kiss, and J.L. Hudson, " Experiments on arrays of globally coupled chaotic electrochemical oscillators: Synchronization and clustering", Chaos 10 (2000) 248

[32] I.Z. Kiss, Y.Zhai, and J.L. Hudson, "Collective dynamics of chaotic chemical oscillators and the law of large numbers", Phys. Rev. Lett. 88(2002)238301

[33] Yanagita, T. and K. Kaneko, "Rayleigh-Bénard convection", Physica D 82 (1995) 288.

[34] Sano M, Kokubo H, Janiaud B, et al. "Phase wave in a cellular structure", Prog Theor Phys 90: 1-34 1993

[35] Itoh H, Kimoto M, Chaotic itinerancy with preferred transition routes appearing in an atmospheric model, PHYSICA D 109: (3-4) 274-292 NOV 151997

[36] Itoh H, Kimoto M Weather regimes, low-frequency oscillations, and principal patterns of variability: A perspective of extratropical lowfrequency variability, Atmospheric Sciences 56: 2684-2705 1999 
[37] K. Kaneko, "Relevance of Clustering to Biological Networks", Physica $75 \mathrm{D}(1994) 55$

[38] K. Kaneko, Recursiveness, Switching, and Fluctuations in a Replicating Catalytic Network, Phys. Rev. E., in press

[39] C. Furusawa and K. Kaneko, Theory of Robustness of Irreversible Differentiation in a Stem Cell System: Chaos Hypothesis, J. Theor. Biol. (2001) 209 (2001) 395-416

[40] C. Gray, A. K. Engel, P. Koenig and W. Singer, Synchronization of oscillatory neural responses in cat striate cortex: Temporal properties, VISUAL NEUROSCIENCE 8 337-347 1992.

[41] Tsuda,I, Fujii,H., Tadokoro, S. and T. Yasuoka, Chaotic itinerancy in cortical gap junction systems and its functional significance, submitted to J. of Integrative Neurosciences, June, 2003.

[42] Tsuda, I., Toward an interpretation of dynamic neural activity in terms of chaotic dynamical systems, BEHAVIORAL AND BRAIN SCIENCES 24: (5) 793-847 2001; The plausibility of a chaotic brain theory, ibid. as a response.

[43] E. Akiyama and K. Kaneko, "Evolution of Cooperation, Differentiation, Complexity, and Diversity in an Iterated Three-person Game", Artificial Life 2 (1996) 293-304

[44] Nicolis, G. and I. Prigogine, Self--Organization in Nonequilibrium Systems (Wiley, 1977)

[45] Haken, H., Synergetics (Springer-Verlag, Berlin, Heidelberg, 1979).

[46] Kaneko, K. and T. Ikegami, "Homeochaos: Dynamics stability of a symbiotic network with population dynamics and evolving mutation rates", Physica D 56 (1992) 406.

[47] Matsumoto, K. and Tsuda, I. , Information theoretical approach to noisy dynamics, JOURNAL OF PHYSICS A:Math.Gen.18 3561-3566 1985 .

[48] Matsumoto, K. and Tsuda, I. , Extended information in onedimensional maps, Physica 26D 347-357 1987. 
[49] Matsumoto, K. and Tsuda, I., Calculation of information flow rate from mutual information, JOURNAL OF PHYSICS A:Math.Gen.21 1405-1414 1988.

[50] Tsuda, I., Koerner, E. and Shimizu, H., Memory dynamics in asynchronous neural networks, PROGRESS OF THEORETICAL PHYSICS 78 51-71 1987.

[51] Nicolis, J. S. and Tsuda, I., Mathematical description of brain dynamics in perception and action, JOURNAL OF CONSCIOUSNESS STUDIES, 6 215-228 1999.

[52] Freeman, W. J., SOCIETIES OF BRAINS: A study in the neuroscience of love and hate. (Lawrence Erlbaum Associates, Inc., Publ., Hillsdale, New Jersey, 1995);

Freeman, W. J., HOW BRAINS MAKE UP THEIR MINDS. (A Phoenix Papaer back, London, 1999) 